\title{
Using Numerical Simulations to Explore Top-Mounted Propulsion on a Conceptual Commercial Supersonic Transport Aircraft
}

\author{
David J. Friedlander ${ }^{1}$ \\ NASA Glenn Research Center, Cleveland, OH, 44135
}

\begin{abstract}
Reynolds Averaged Navier-Stokes (RANS) simulations were performed on an $\mathrm{N}+2$ conceptual commercial supersonic transport aircraft to explore the possibility of relocating the engines from below the wings to above the wings in order to capitalize upon potential noise shielding benefits. The simulations focused on the feasibility of the top-mounted propulsion configuration in terms of inlet performance and flow separation around the nacelles at climbout conditions for both $0^{\circ}$ and $8^{\circ}$ angle of attack. The results showed overall good inlet performance and little separation around the nacelles. The results were comparable to the engines in their original underwing configuration.
\end{abstract}

\section{Nomenclature}

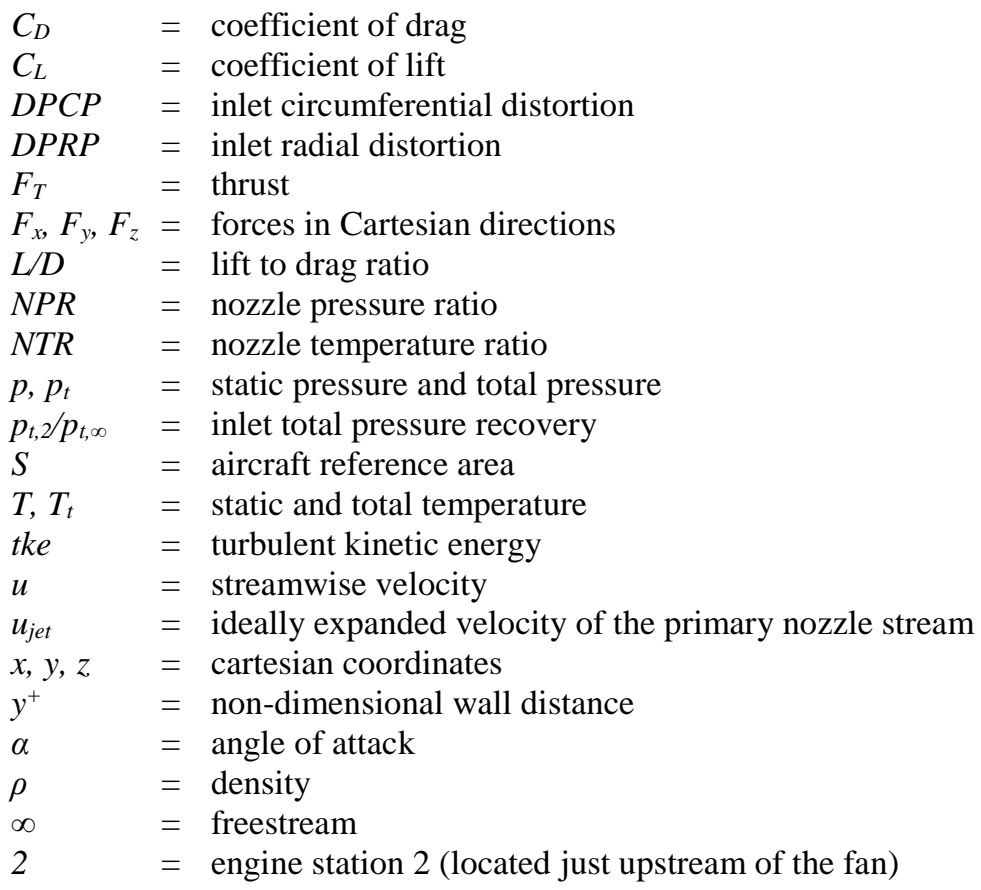

\section{Introduction}

COMMERCIAL aircraft are currently forbidden from flying supersonically overland in the United States, per regulations dating back to the $1970 \mathrm{~s}^{1,2}$. Despite this, there is an interest within the aviation community to see the resurgence of commercial supersonic transport aircraft into the national airspace. The NASA Aeronautics Research Mission Directorate has incorporated this desire into their Strategic Implementation Plan, with one of its goals being to aid in the "introduction of affordable, low-boom, low-noise, and low-emission [commercial] supersonic transports"

${ }^{1}$ Aerospace Engineer, Inlets and Nozzles Branch, 21000 Brookpark Road, MS 5-12, AIAA Member. 
by $2035^{3}$. The Lockheed Martin LM1044-3b, a conceptual N+2 commercial supersonic transport aircraft ${ }^{4}$ shown in Fig. 1, has been used in prior studies to better understand jet-surface interactions and to explore jet noise reduction techniques $^{5-8}$. One such technique was to move the engines from underneath the wings to above the wings, which would allow for potential jet noise shielding relative to ground observers. While a recent test was conducted at the NASA Glenn Research Center's AeroAcoustic Propulsion Laboratory to help quantify this acoustic benefit, preliminary Reynolds Averaged Navier-Stokes (RANS) simulations were performed prior to the test to assess whether this concept would be feasible: i.e. would flow separation occur around the nacelles? Would inlet performance for top-mounted propulsion at take-off conditions suffer? This paper presents the results of these simulations for a topmounted propulsion version of the conceptual LM1044-3b transport aircraft.

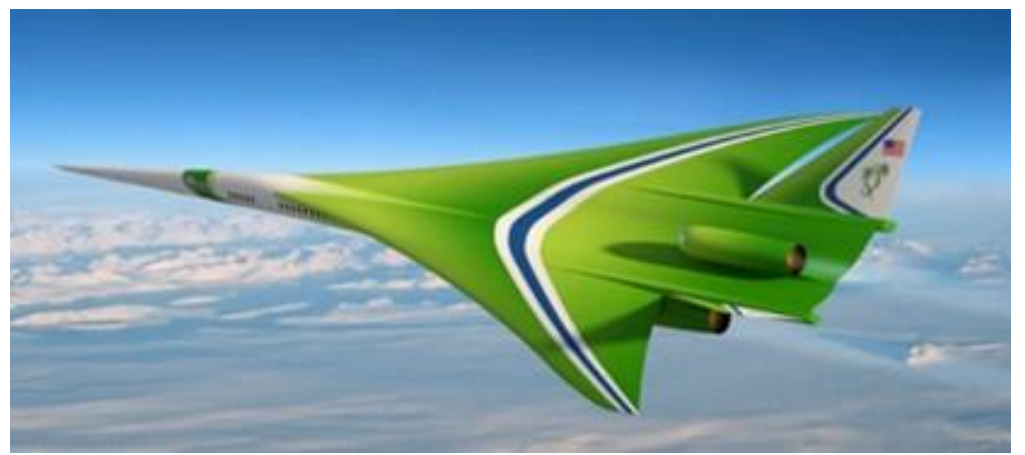

Figure 1. LM1044-3b conceptual aircraft by Lockheed Martin4.

\section{Geometry and Numerical Modeling}

\section{A. Geometry and Grid Representation}

The LM1044-3b is a conceptual supersonic transport aircraft with three engines: outboard engines mounted underneath each wing and one top-mounted center engine. Each engine comprises of an external compression, axisymmetric spike inlet with auxiliary doors and a three-stream inverted velocity profile nozzle. The LM1044-3b geometry was used as a starting point from which the outboard engines were moved from underneath the wing to above the wing. The new top-mounted outboard engine locations were identical to a previous study ${ }^{8}$ with the original and final locations of the outboard engines shown in Fig. 2. Due to the top-mounted outboard engine pylon geometry being unavailable from that previous study, a simple pylon, shown in Fig.3, was designed to attach the outboard engines to the airframe. The inboard side of the pylon bottoms, when viewed from the top, were designed to the form of a quadratic contour, which was symmetric about the mid-point of the pylons. The outboard side of the pylon bottoms, when viewed from the top, were simply merged with the aircraft airframe. For comparison purposes, the outboard engine pylon used for the under-the-wing configuration is also shown in Fig. 3. The reader is cautioned that there was no optimization involved in the design of the top-mounted outboard engine pylons. The resulting new topmounted propulsion aircraft geometry, referred to in this paper as the LM1044-TMP, is shown in Fig. 4.

An unstructured viscous grid was constructed using the Pointwise grid generation software ${ }^{9}$ to represent the LM1044-TMP geometry. Due to symmetry, only half of the aircraft geometry was modeled. Additionally, the support struts within the inlets were not modeled. The initial grid consisted of 14.6 million nodes with examples of the surface grid shown in Fig. 5. The viscous grid layers were spaced such that there was a $y^{+}<2$ for most of the surface grid with pockets along the pylon edges that had $y^{+}$values as high as 3.2. The volume grid also included node clustering around the plume regions, with node spacing of 2 inches. An initial plume grid study showed lower than expected values of turbulent kinetic energy ( $t k e$ ) within the plume regions based on earlier simulations of a similar isolated nozzle configuration ${ }^{7}$. The spacing between nodes within the plume regions was subsequently halved, with results shown in Fig 6. The initial grid with the "refined" plume grid, totaling 19.2 million nodes, would become the baseline grid for the simulations presented in this paper. 

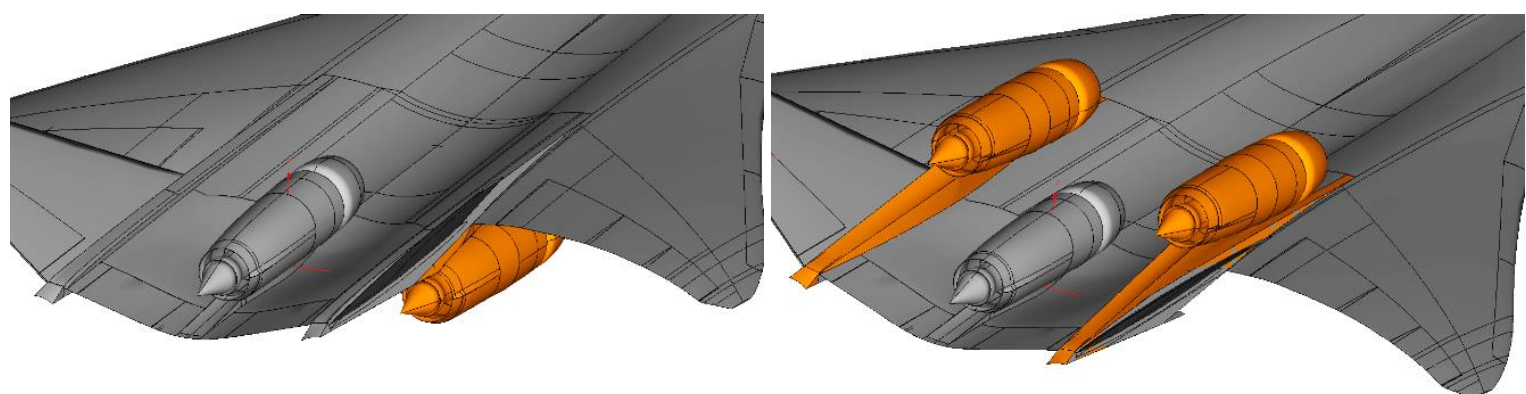

Figure 2. The outboard engines were relocated from under the wing (left) to above the wing (right).
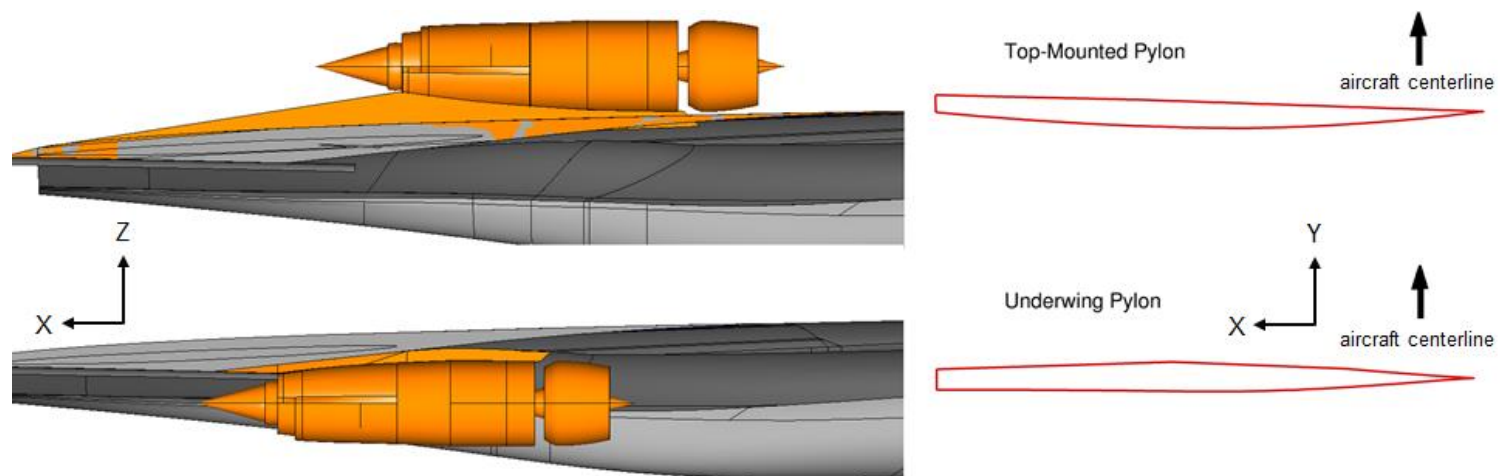

Figure 3. An outboard engine pylon outboard view (left) and corresponding bottom contour (right) for both the top-mounted and under-the-wing configurations. Note that the center engine, tails, and wings have been removed from the outboard views for clarity and that the bottom contours are not to the same scale as the outboard views.

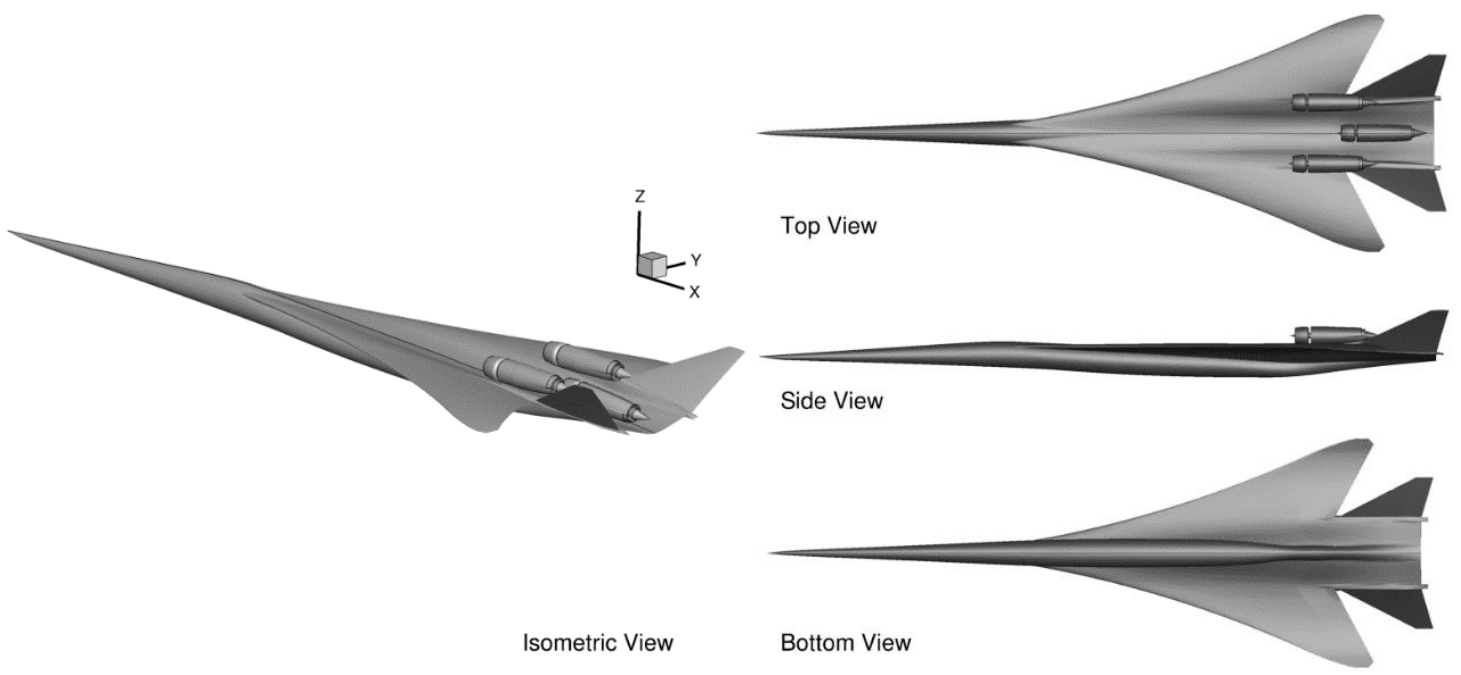

Figure 4. LM1044-TMP geometry 

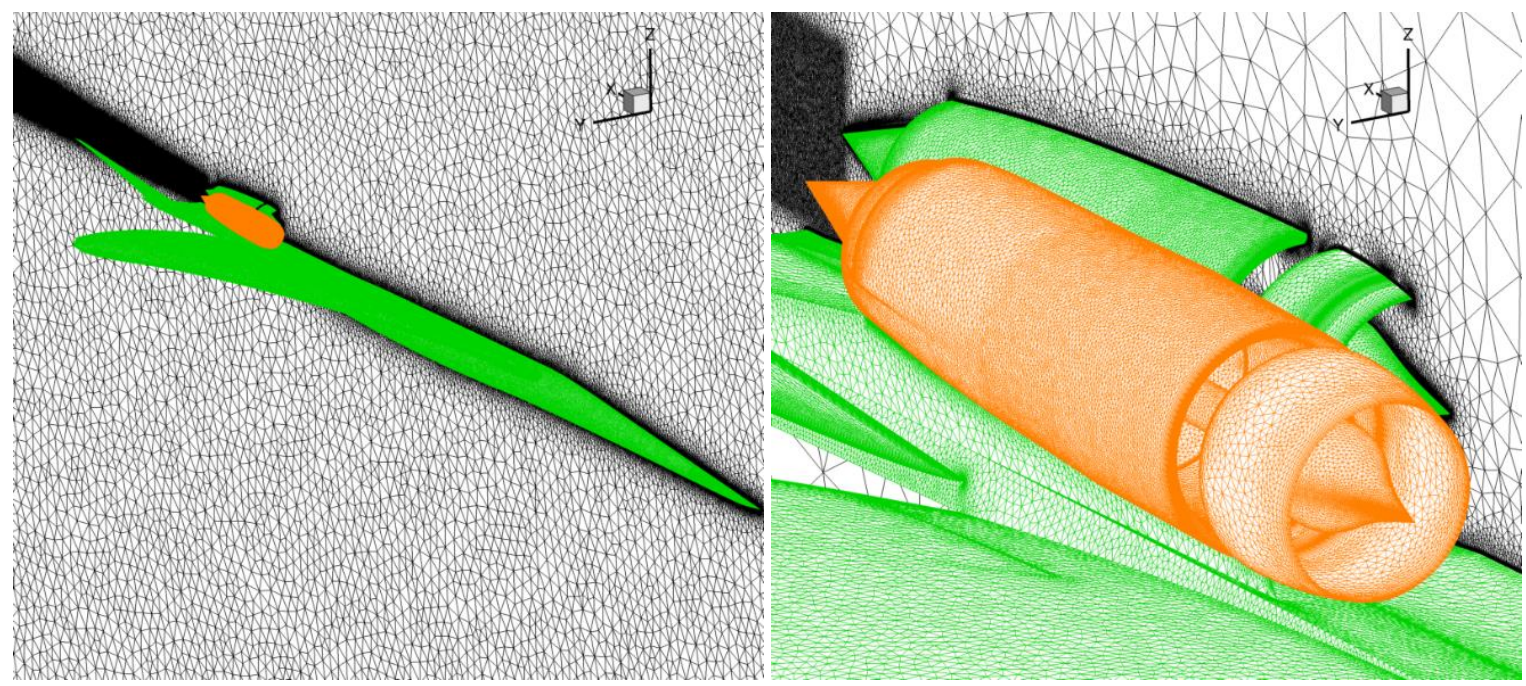

Figure 5. Surface grid around the aircraft (left) and around the engine nacelles (right).
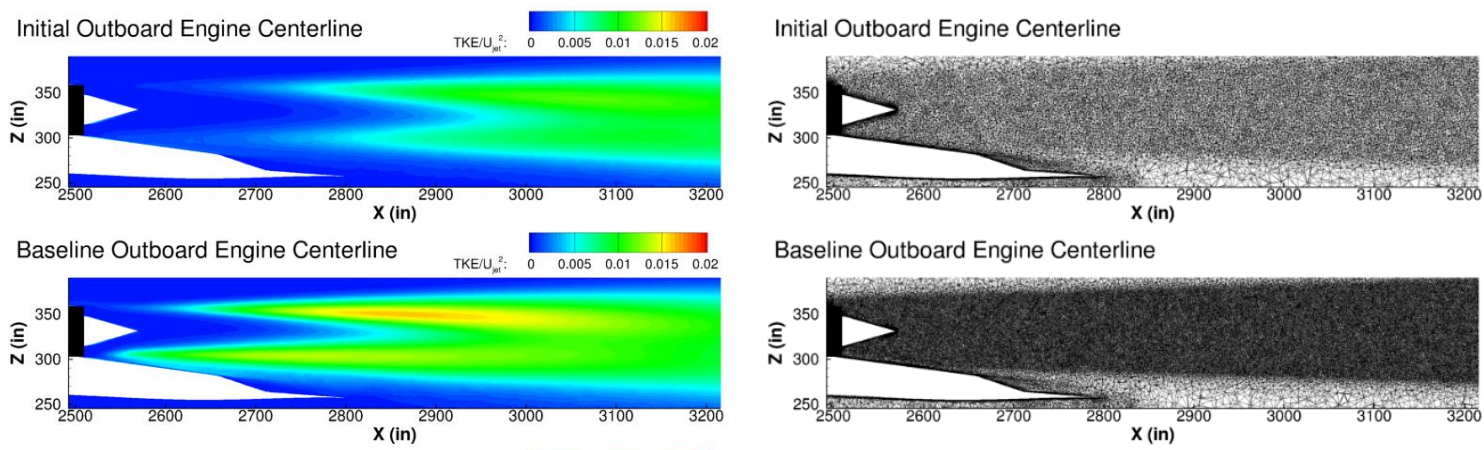

Baseline Outboard Engine Centerline
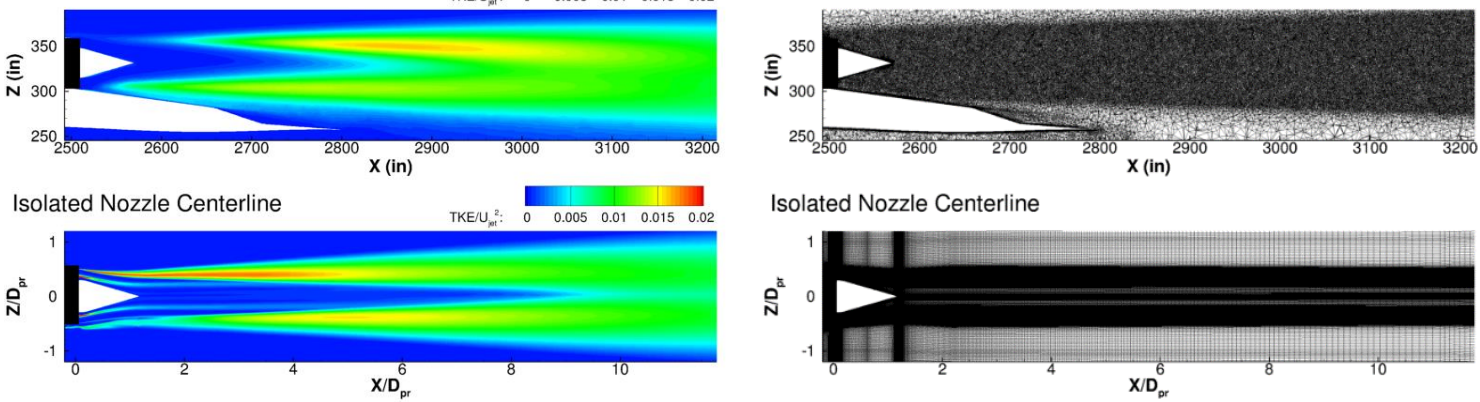

Isolated Nozzle Centerline

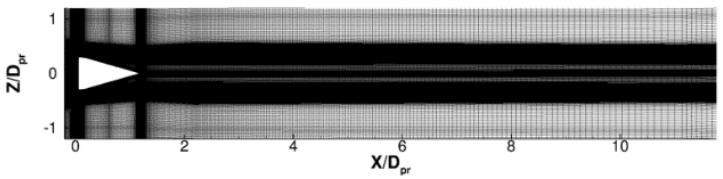

Figure 6. Contour plots of $t k e$ of the outboard engine plume region for the initial grid clustering (top), the final refined plume grid clustering (middle), and the isolated nozzle grid $^{7}$ (bottom).

\section{B. Flow Solver and Boundary Conditions}

The FUN3D ${ }^{10}$ flow solver was used for all simulations due to its ability to handle unstructured grid representations of complex geometries. FUN3D is a node-based, finite volume Navier-Stokes solver and can solve both compressible and incompressible flow problems. The simulations used the Roe flux-difference splitting scheme ${ }^{11}$ with the Van Leer flux-vector splitting scheme ${ }^{12}$ for the left hand side. All simulations used Menter's Shear Stress Transport (SST) turbulence model ${ }^{13}$.

Simulations were run at climb-out conditions at both $0^{\circ}$ and $8^{\circ}$ angle of attack. Table 1 outlines the freestream conditions while Table 2 lists the nozzle inflow boundary conditions. The inlet outflow boundary condition was set to $860 \mathrm{lbm} / \mathrm{s}$. All engines used the same boundary conditions.

Table 1. Freestream conditions.

\begin{tabular}{|c|c|}
\hline Mach Number & 0.3 \\
\hline Pressure & $14.3 \mathrm{psi}$ \\
\hline Temperature & $530^{\circ} \mathrm{R}$ \\
\hline
\end{tabular}


Table 2. Nozzle inflow conditions.

\begin{tabular}{|c|c|c|}
\hline Nozzle Stream & NPR & NTR \\
\hline Inner & 1.78 & 1.245 \\
\hline Primary & 2.00 & 1.887 \\
\hline Buffer & 1.78 & 1.245 \\
\hline
\end{tabular}

\section{Results}

\section{A. Grid Refinement Study}

An initial grid refinement study was performed, using the baseline grid as the starting point. Grid refinement consisted of uniformly increasing the node count on the surface connectors by a factor of 1.25 and subsequently decreasing the initial spacing off of the viscous surfaces by a factor of 1.25 . This resulted in a volume grid with 32.3 million nodes. For simplicity, only the $0^{\circ}$ angle of attack case was simulated for the initial grid refinement study.

Several different parameters were chosen to help quantify grid independence. First, a qualitative approach was chosen to examine the grid sensitivity in the plume regions by plotting the tke normalized by the ideally expanded primary stream jet velocity. Engine centerline cuts are shown in Fig. 7, which upon inspection, show that the baseline grid is not grid independent in these regions. A quantitative approach was chosen for the remaining parameters, starting with the lift coefficient, drag coefficient, and the lift-to-drag ratio of the aircraft. These were computed in order to determine the grid sensitivity to the overall aircraft surface and are displayed in Table 3 . The coefficients of lift and drag were defined as follows:

$$
\begin{aligned}
& C_{L}=\frac{\left(-F_{x} \times \sin \alpha\right)+\left(F_{z} \times \cos \alpha\right)}{0.5 \times \rho_{\infty} \times u_{\infty}^{2} \times S} \\
& C_{D}=\frac{\left(F_{x} \times \cos \alpha\right)+\left(F_{z} \times \sin \alpha\right)}{0.5 \times \rho_{\infty} \times u_{\infty}^{2} \times S}
\end{aligned}
$$

where

$$
\begin{gathered}
\rho_{\infty}=0.00226 \mathrm{slug} / \mathrm{ft}^{3} \\
u_{\infty}=338.5 \mathrm{ft} / \mathrm{s}^{2} \\
S=3600 \mathrm{ft}^{2}
\end{gathered}
$$

Note that the forces $F_{x}$ and $F_{z}$ do not include the forces on the engines nor the engine nacelles and the reference surface area $S$ was provided by Morgenstern et. al. ${ }^{4}$ Based on the results in Table 3, it can be shown that the lift coefficient, drag coefficient, and the lift-to-drag ratio are changing by at most $1.6 \%$ relative to the baseline grid values. In order to get a sense of the grid sensitivity in the inlet regions, the average total pressure recovery and the ARP 1420 distortion parameters ${ }^{14} D P C P$ and $D P R P$ were computed at the aerodynamic interface planes (AIPs) for both the outboard and center engine inlets. These parameters, displayed in Table 4 for the outboard engine inlet and Table 5 for the center engine inlet, show that while the average total pressure recovery is changing by only $0.08 \%$, the average inlet distortions are changing by as much as $20 \%$ compared to the baseline grid values. Finally, the thrust for each engine was computed to get a quantitative idea of the grid sensitivity in the nozzle regions, with the percent difference in thrust between the two grids shown in Table 6. Based on this data, it is shown that the thrust is changing marginally between the two grids by at most $0.32 \%$, which suggests grid convergence in the nozzle regions. Even though the refined grid is not shown to be grid independent in some regions, it was determined that it was more grid independent than the baseline grid and thus the remainder of the simulations were performed strictly on the refined grid.

Table 3. Lift coefficient, drag coefficient, and the lift-to-drag ratio for $\alpha=0^{\circ}$.

\begin{tabular}{|c|c|c|c|}
\cline { 2 - 4 } \multicolumn{1}{c|}{} & Baseline Grid & Refined Grid & \% Difference \\
\hline $\boldsymbol{C}_{\boldsymbol{L}}$ & 0.04937 & 0.04963 & 0.5 \\
\hline $\boldsymbol{C}_{\boldsymbol{D}}$ & 0.00691 & 0.00683 & -1.1 \\
\hline $\boldsymbol{L} / \boldsymbol{D}$ & 7.144 & 7.261 & 1.6 \\
\hline
\end{tabular}


Table 4. Average total pressure recovery and inlet distortion for the outboard engine inlet for $\alpha=0^{\circ}$.

\begin{tabular}{|l|c|c|c|}
\cline { 2 - 4 } \multicolumn{1}{c|}{} & Baseline Grid & Refined Grid & \% Difference \\
\hline $\boldsymbol{p}_{t, 2} / \boldsymbol{p}_{t, \infty}$ & 0.994 & 0.995 & 0.1 \\
\hline $\boldsymbol{D P C P}$ & 0.0011 & 0.0010 & -9.1 \\
\hline $\boldsymbol{D P R P}$ & 0.0060 & 0.0056 & -6.7 \\
\hline
\end{tabular}

Table 5. Average total pressure recovery and inlet distortion for the center engine inlet for $\alpha=0^{\circ}$.

\begin{tabular}{|l|c|c|c|}
\cline { 2 - 4 } \multicolumn{1}{c|}{} & Baseline Grid & Refined Grid & \% Difference \\
\hline $\boldsymbol{p}_{t, 2} / \boldsymbol{p}_{\boldsymbol{t}, \infty}$ & 0.993 & 0.993 & 0.0 \\
\hline $\boldsymbol{D P C P}$ & 0.0049 & 0.0047 & -4.1 \\
\hline $\boldsymbol{D P R P}$ & 0.0055 & 0.0066 & 20.0 \\
\hline
\end{tabular}

Table 6. Percent difference $\left(F_{T, \text { refined }}-F_{T, \text { initial }}\right)$ in thrust for $\alpha=0^{\circ}$.

\begin{tabular}{|r|c|}
\cline { 2 - 2 } \multicolumn{1}{c|}{} & \% Difference \\
\hline Outboard Engine & -0.2 \\
\hline Center Engine & 0.3 \\
\hline
\end{tabular}
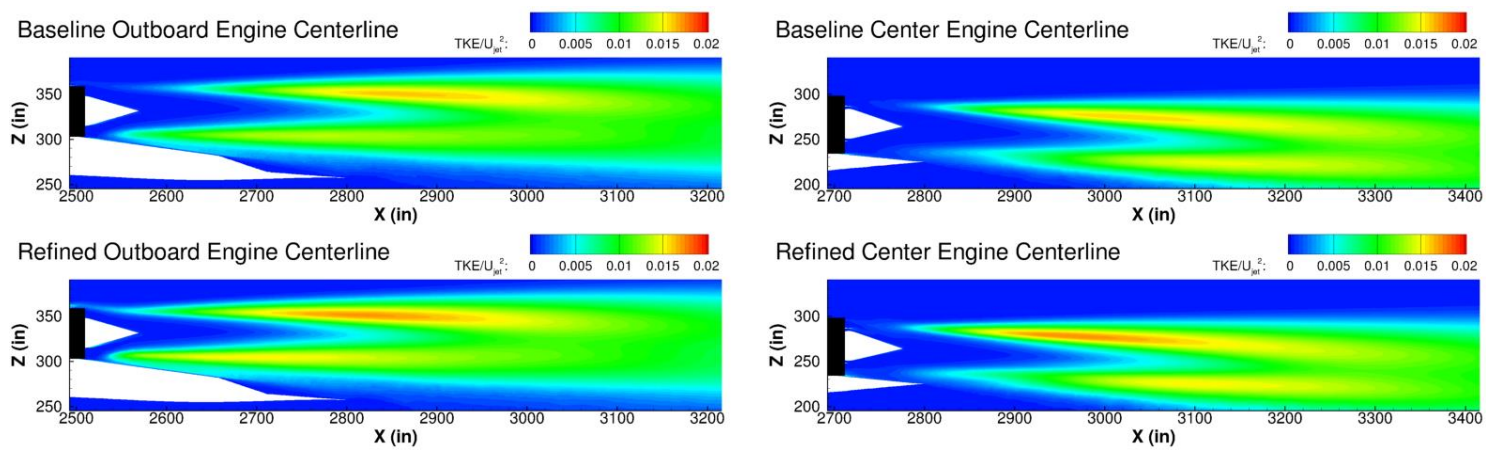

Figure 7. tke contour plots for the baseline grid (top) and the refined grid (bottom), for the outboard engine plume region (left) and the center engine plume region (right) for $\alpha=0^{\circ}$.

\section{B. Inlets}

Figure 8 shows iso-surfaces of negative $u$ velocity, colored by the non-dimensional $u$ velocity, around the inlet nacelles. The iso-surface was defined at a non-dimensional $u$ velocity of -0.0001 . The lack of red regions in the figure implies that that there is very little separation outside of the inlets around their respective nacelles. For another perspective, Fig. 9 shows the $u$ velocity contours non-dimensionalized by the freestream $u$ velocity for the outboard engine centerline at both $0^{\circ}$ and $8^{\circ}$ angle of attack. Based on the figure, there is very little separation within the inlet with exception of inside the upper lip of the nacelle. Also there is very little difference between the two angles of attack. Figure 10 shows $u$ velocity contours at various axial stations for $0^{\circ}$ and $8^{\circ}$ angles of attack with axial station references shown in Fig. 11. Just like the engine centerline plot, there is virtually no separation present within the inlet. There is some sensitivity to angle of attack downstream of the auxiliary doors (downstream of $x=2260$ ) with slightly lower velocity flow within the inlet at $8^{\circ}$ angle of attack compared to $0^{\circ}$ angle of attack. The lack of separation internal to and external of the inlets indicates that there is a reduced risk of extra noise being generated in and around the inlet nacelles.

Aside from looking at potential sources of flow separation in/around the inlets, inlet performance data was also computed. Figure 12 shows the 40-probe total pressure recovery contour plots for both the center and outboard engine inlets. It can be seen that both inlets have a reduced total pressure recovery as the angle of attack is increased. This is further supported by the computed average total pressure recovery at the AIP, shown in Fig. 13. Figure 14 shows the ARP 1420 distortion parameters for both the outboard and center engine inlets. It can be seen that for the outboard engine inlet, the circumferential and radial distortion both increase with the higher angle of attack. However, this trend is reversed for the center engine inlet. 
For additional comparisons with the underwing configuration, the LM1044-TMP was run at two additional angles of attack: $6^{\circ}$ and $9^{\circ}$. Based on Fig. 13 and Fig. 14, it can be shown that the computed inlet performance data for the top-mounted configuration is similar to the under-the-wing configuration, with slight improvements in the average total pressure recovery (by as much as $0.2 \%$ ) and inlet distortion (by as much as $88.1 \%$ for the circumferential distortion and $38.4 \%$ for the radial distortion). These simulations suggest that there is very little inlet performance penalty for mounting the outboard engines above the wing at climb-out.

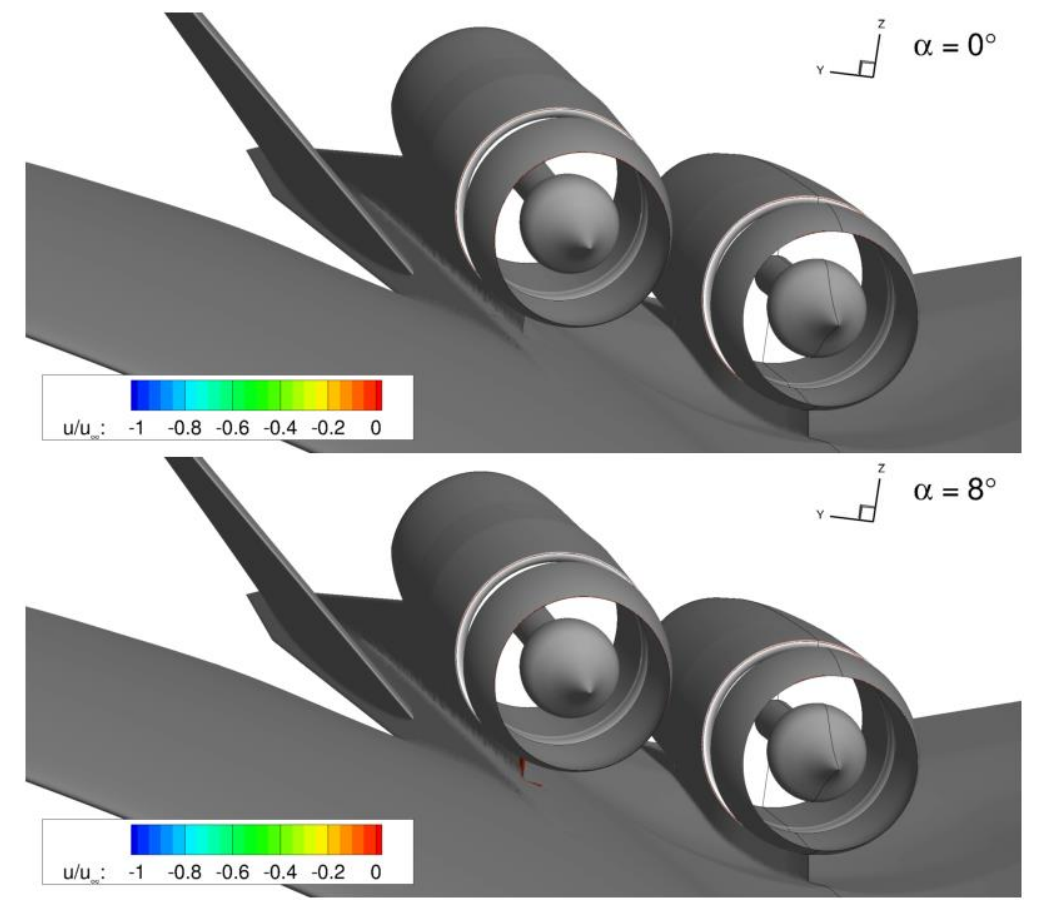

Figure 8. Iso-surfaces of negative $u$ velocity around the inlet end of the nacelles for $\alpha=0^{\circ}$ (top) and $\alpha=8^{\circ}$ (bottom). Lack of red regions implies very little separation.
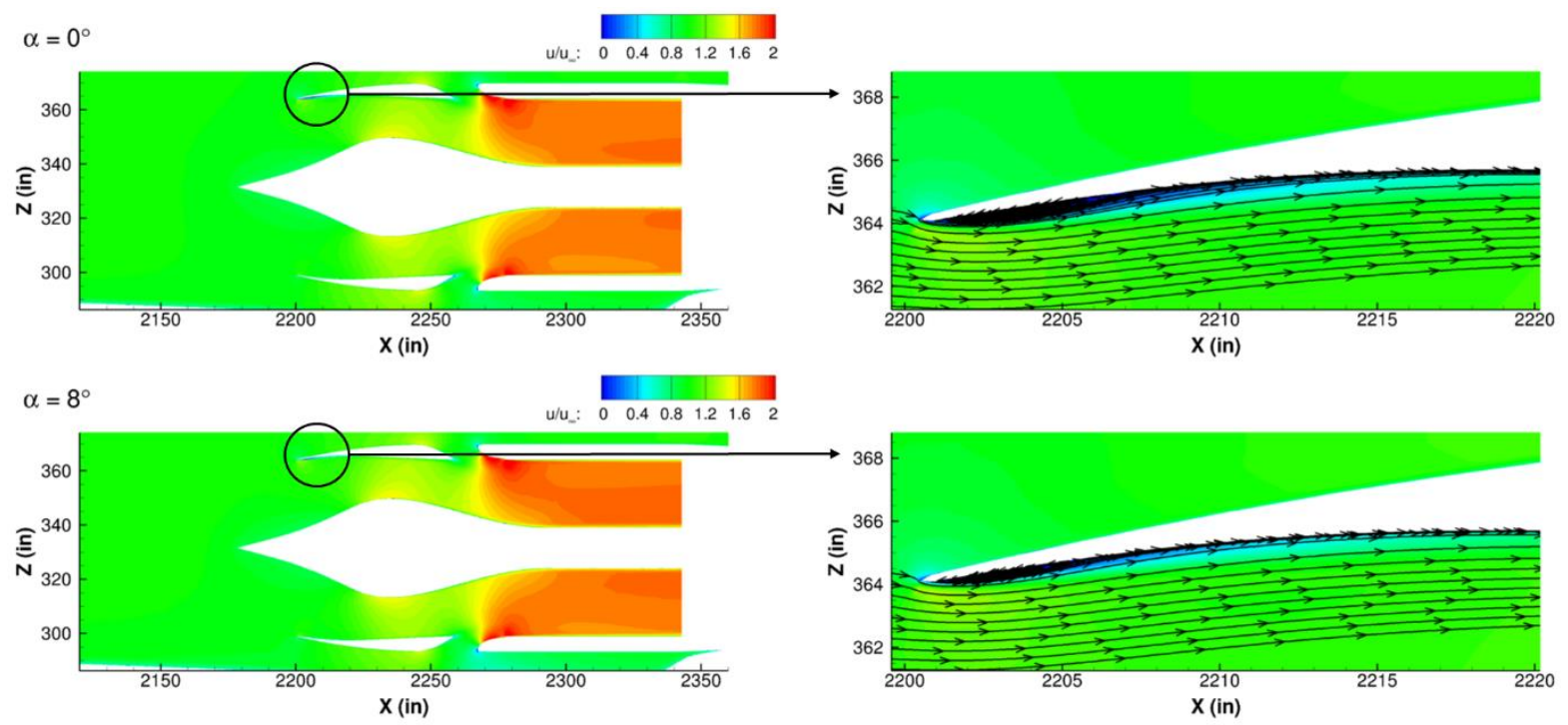

Figure 9. $u$ velocity contour of the outboard engine centerline for $\alpha=0^{\circ}$ (top) and $\alpha=8^{\circ}$ (bottom). Angle of attack inflow distortion has little impact on the inlet flow. 


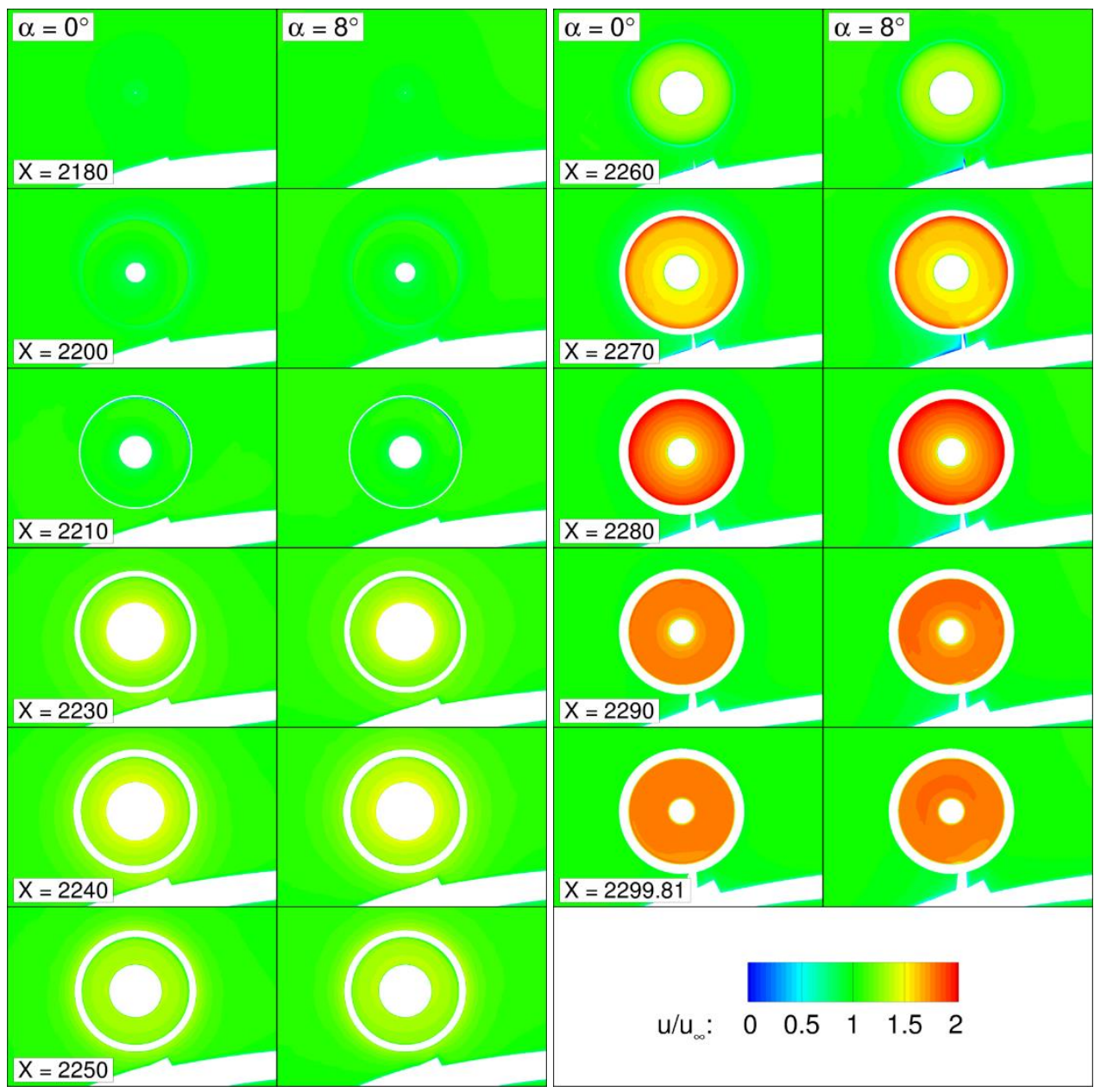

Figure 10. $u$ velocity contours of the outboard engine inlet. Axial stations are in inches.

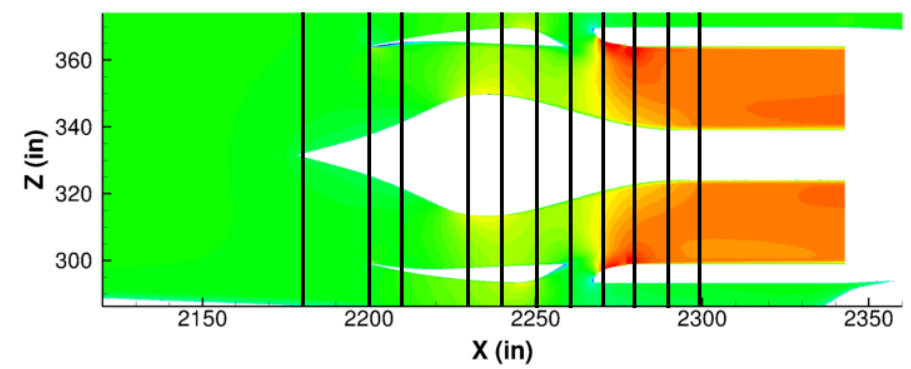

Figure 11. Reference axial stations (vertical black lines) for the outboard engine inlet. The most downstream axial station is the AIP $(x=2299.81)$. 

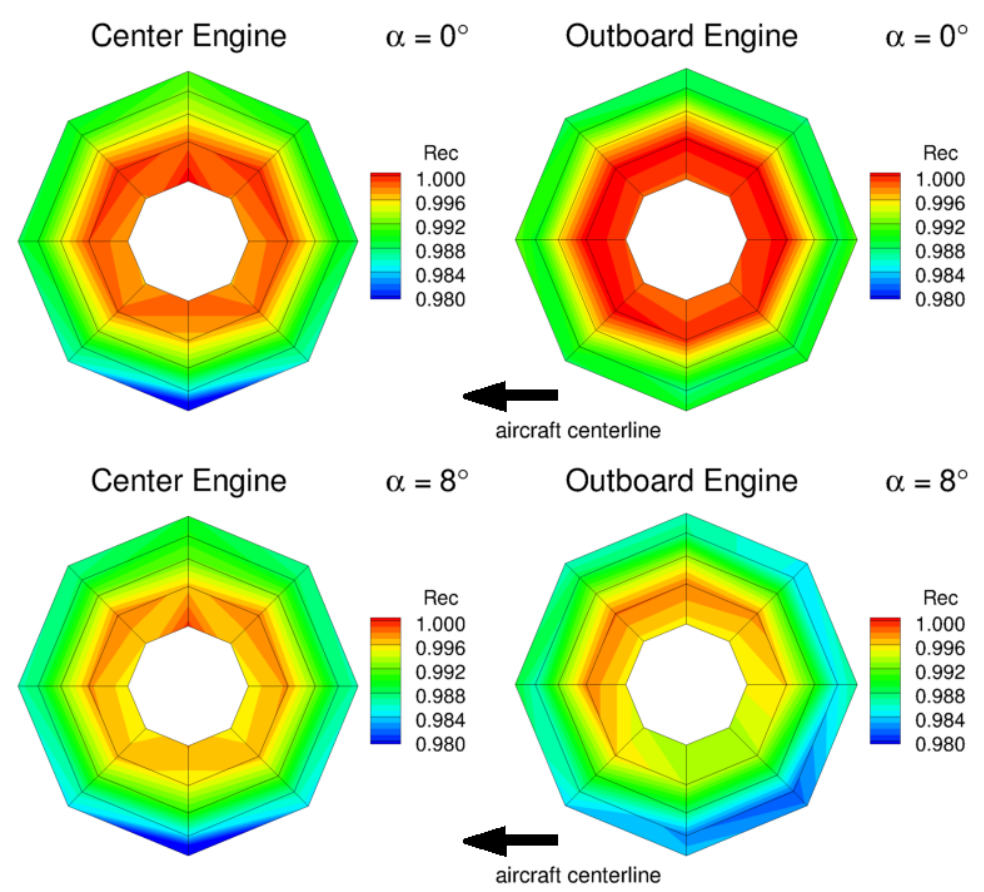

Figure 12. Top-mounted engine configuration (LM1044-TMP) 40-probe total pressure recovery plots for the center engine inlet (left) and outboard engine inlet (right) for $\alpha=0^{\circ}$ (top) and $\alpha=8^{\circ}$ (bottom).

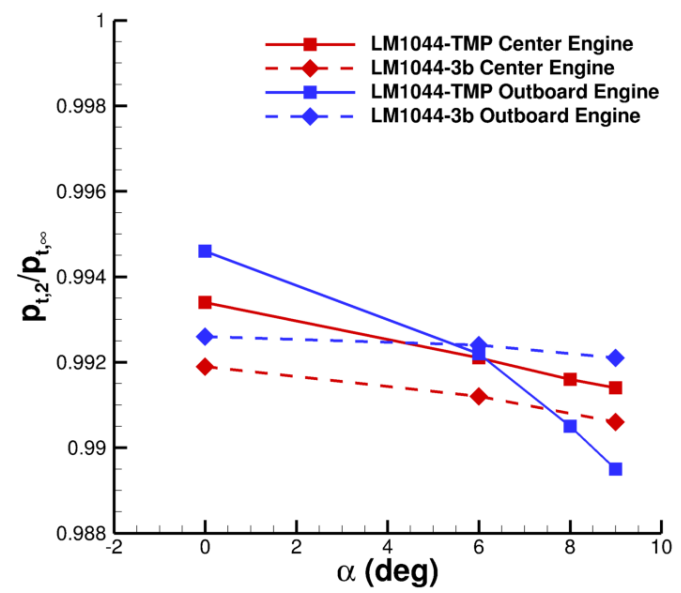

Figure 13. Average total pressure recovery as a function of angle of attack. Top-mounted engines have better average total pressure recovery below $\alpha=6^{\circ}$. 

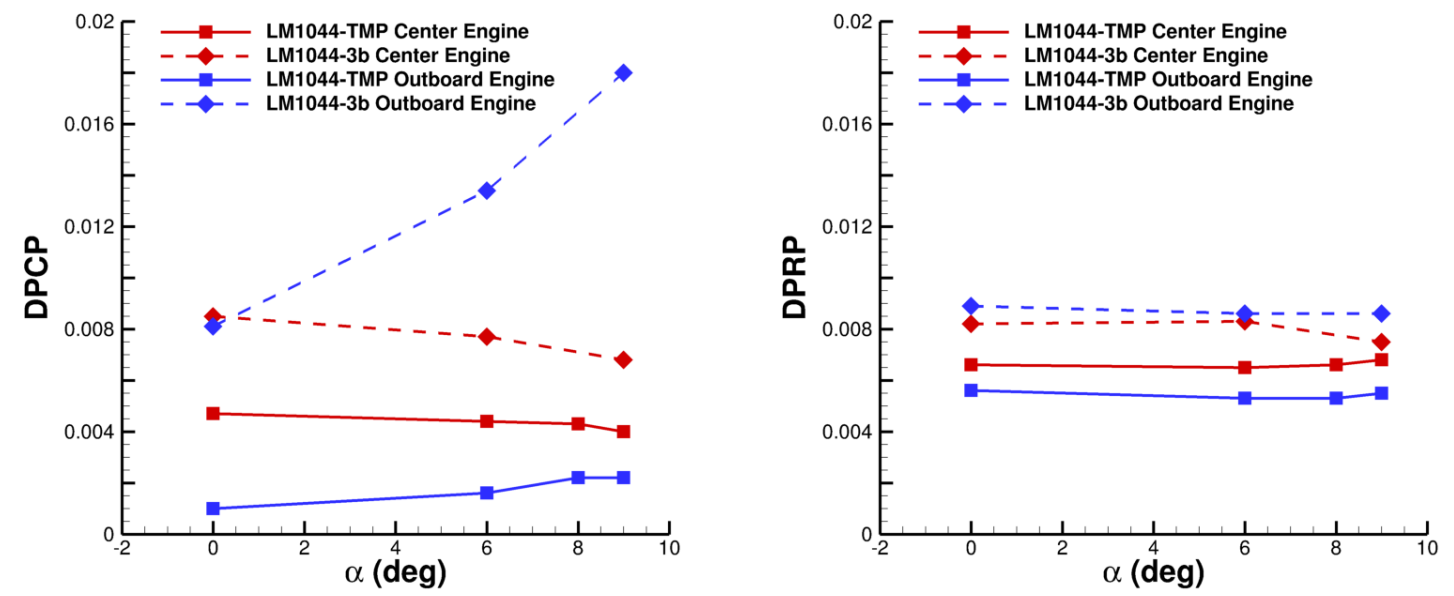

Figure 14. Circumferential (left) and radial (right) distortion as functions of angle of attack. Topmounted engines have reduced distortion factors.

\section{Nozzles}

Figure 15 shows iso-surfaces of negative $u$ velocity, colored by the non-dimensional $u$ velocity, around the nozzle nacelles and aft deck. The iso-surface was defined at a non-dimensional $u$ velocity of -0.0001 . Just like what was shown in Fig. 8 around the inlet nacelles, the lack of red regions in the figure shows that there is very little separation outside the nozzle and aft deck areas and the separations (represented by the red regions) are fairly consistent between the two angles of attack. Figures 16 shows tke contour plots non-dimensionalized by the primary stream ideally expanded jet velocity at various axial stations around the outboard engine nozzles for $0^{\circ}$ and $8^{\circ}$ angle of attack. See Fig. 17 for axial station references. It can be seen that there is very little difference in the tke contour plots between the two angles of attack and that there are no pockets of high tke around the outboard engine nozzle. This, coupled with very little separation outside the nozzles, suggests that there is a reduced risk of extra noise being generated around the engine nozzles and aft deck. 

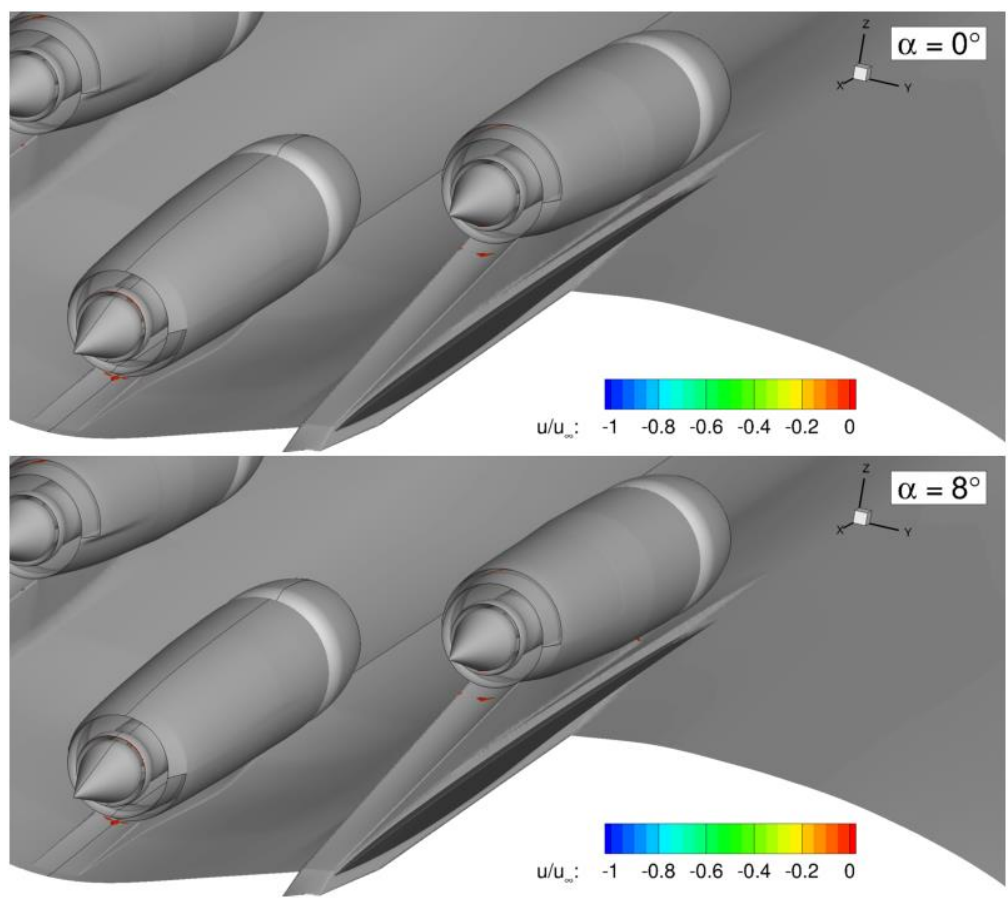

Figure 15. Iso-surfaces of negative $u$ velocity around the nozzle end of the nacelles for $\alpha=0^{\circ}$ (top) and $\alpha=8^{\circ}$ (bottom). Lack of red regions implies very little separation.

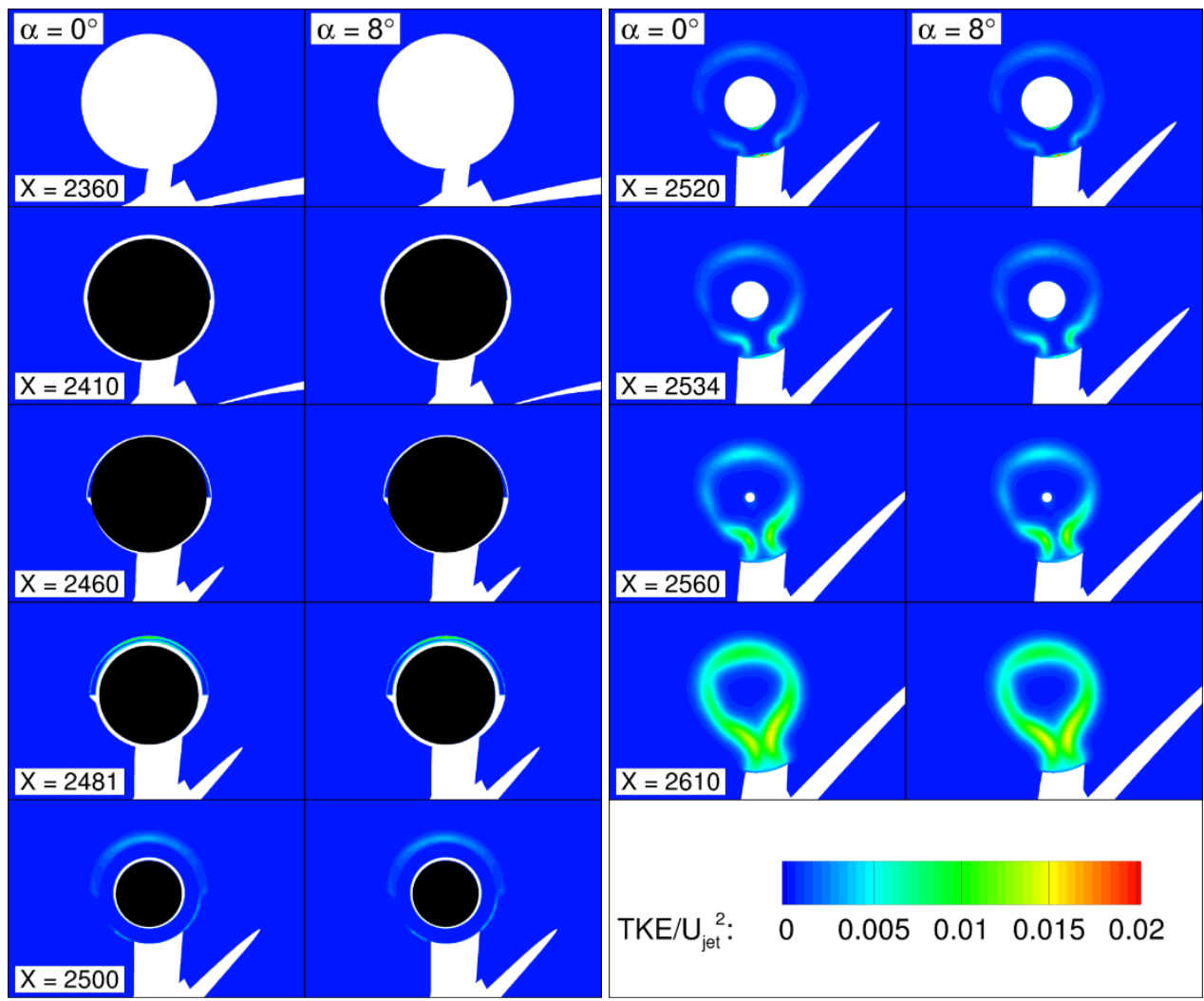

Figure 16. tke contours of the outboard engine nozzle. Axial stations are in inches. 


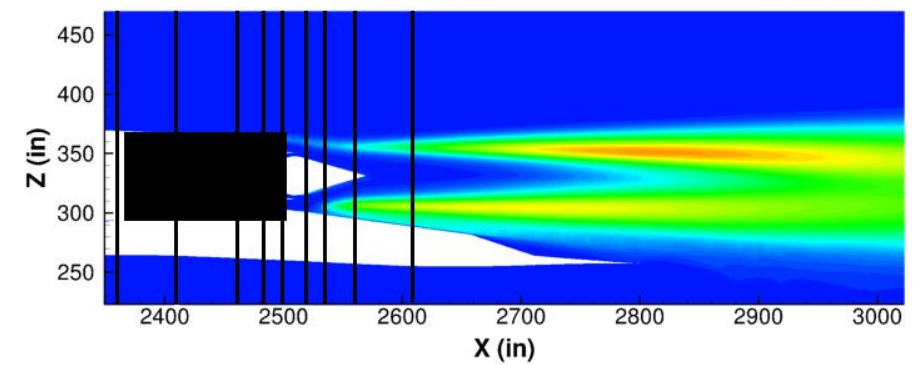

Figure 17. Reference axial stations (vertical black lines) for the outboard engine nozzle.

\section{Conclusions/Recommendations}

To conclude, RANS simulations of the LM1044-3b conceptual supersonic transport aircraft were performed with the outboard engines mounted above the wing instead of beneath the wing. The simulations were completed at climbout conditions at both $0^{\circ}$ and $8^{\circ}$ angle of attack. The results showed little sensitivity to the angle of attack with respect to flow separation-based potential noise sources. In addition, these simulations showed that there is no inlet performance penalty for mounting the outboard engines above the wing, as the computed inlet distortion and total pressure recovery were comparable to that of the initial under-the-wing configuration. One recommendation for an additional study would be to assess the possible fan/inlet noise implications of this top-mounted propulsion system. A final recommendation would be to apply the lessons learned in simulating this configuration to future $\mathrm{N}+2$ commercial supersonic transport conceptual aircraft designs.

\section{Acknowledgments}

The author would like to thank the NASA Advanced Air Vehicles Program, Commercial Supersonic Technology Project for support with this research. The author would also like to thank Vance Dippold for giving guidance throughout the project and for providing the initial surface grid and John Slater for providing a computer code to compute the ARP 1420 distortion parameters.

\section{References}

1“14 CFR Parts 36 and 91: Civil Supersonic Airplane Noise Type Certification Standards and Operating Rules,” FAA Supersonic Aircraft Noise, URL:

https://www.faa.gov/about/office_org/headquarters_offices/apl/noise_emissions/supersonic_aircraft_noise/media/noise_policy_o n_supersonics.pdf [cited 30 April 2018].

${ }^{2}$ Civil Aircraft Sonic Boom, 14 C.F.R. § 91.8172017.

"NASA Aeronautics Strategic Implementation Plan 2017 Update," NASA Aeronautics Strategic Implementation Plan, URL: https://www.nasa.gov/sites/default/files/atoms/files/sip-2017-03-23-17-high.pdf [cited 30 April 2018].

${ }^{4}$ Morgenstern, J., et al, “Advanced Concept Studies for Supersonic Commercial Transports Entering Service in the 2018-2020 Period, Phase 2," NASA/CR-2015-218719, July 2015.

5ridges, J., Podboy, G. G., and Brown, C. A., "Testing Installed Propulsion for Shielded Exhaust Configurations," AIAA 2016-3042, May 2016.

${ }^{6}$ Brown, C., Podboy, G, and Bridges, J., "Modeling Jet-Surface Interaction Noise for Separate Flow Nozzles," AIAA 20162862, May 2016.

${ }^{7}$ Dippold, V. and Friedlander, D., "Relating a Jet-Surface Interaction Experiment to a Commercial Supersonic Transport Aircraft Using Numerical Simulations," AIAA 2017-1853, January 2017.

${ }^{8}$ Ramakrishnan, K., et al, "Evaluation of Low Noise Integration Concepts and Propulsion Technologies for Future Supersonic Civil Transports," August 2017 (to be published).

${ }^{9}$ Pointwise, Software Package, Version 18.0 Release 3, Pointwise Inc., Fort Worth, TX, 2017.

${ }^{10}$ Biedron, R. T., et al, “FUN3D Manual: 13.1,” NASA/TM-2017-219580, February 2017.

${ }^{11}$ Roe, P. L., “Approximate Riemann Solvers, Parameter Vectors, and Difference Schemes,” Journal of Computational Physics, Vol. 43, 1981, pp. 357-372.

${ }^{12}$ Van Leer, B., "Flux-Vector Splitting for the Euler Equations," ICASE Report 82-30, 1982.

${ }^{13}$ Menter, F. R., "Two-Equation Eddy Viscosity Turbulence Models for Engineering Applications," AIAA Journal, Vol. 32 , No. 8, 1994, pp. 1598-1605.

${ }^{14}$ Society of Automotive Engineers Inc., “Gas Turbine Engine Inlet Flow Distortion Guidelines,” ARP 1420 Rev. B, 2011. 